\title{
Size effects in angle-resolved photoelectron spectroscopy of free rare-gas clusters
}

\author{
D. Rolles, ${ }^{1,2}$ H. Zhang, ${ }^{1}$ Z. D. Pešićc, ${ }^{1,2}$ R. C. Bilodeau, ${ }^{1,2}$ A. Wills, ${ }^{1}$ E. Kukk,${ }^{3}$ B. S. Rude, ${ }^{2}$ G. D. Ackerman, ${ }^{2}$ \\ J. D. Bozek, ${ }^{2,4}$ R. Díez Muiño, ${ }^{5}$ F. J. García de Abajo, ${ }^{6}$ and N. Berrah ${ }^{1}$ \\ ${ }^{1}$ Physics Department, Western Michigan University, Kalamazoo, Michigan 49008, USA \\ ${ }^{2}$ Advanced Light Source, Lawrence Berkeley National Laboratory, Berkeley, California 94720, USA \\ ${ }^{3}$ Department of Physics, University of Turku, 20014 Turku, Finland \\ ${ }^{4}$ Stanford Linear Accelerator Center, Menlo Park, California 94025, USA \\ ${ }^{5}$ Centro de Física de Materiales, Centro Mixto CSIC-UPV/EHU and DIPC, 20018 San Sebastian, Spain \\ ${ }^{6}$ Instituto de Optica, CSIC, 28006 Madrid, Spain \\ (Received 23 May 2006; published 27 March 2007)
}

\begin{abstract}
The photoionization of free Xe clusters is investigated by angle-resolved time-of-flight photoelectron spectroscopy. The measurements probe the evolution of the photoelectron angular distribution parameter as a function of photon energy and cluster size. While the overall photon-energy-dependent behavior of the photoelectrons from the clusters is very similar to that of the free atoms, distinct differences in the angular distribution point at cluster-size-dependent effects. Multiple scattering calculations trace their origin to elastic photoelectron scattering.
\end{abstract}

DOI: 10.1103/PhysRevA.75.031201

PACS number(s): $36.40 . \mathrm{Mr}, 61.46 . \mathrm{Bc}, 73.22 . \mathrm{f}$

Several decades after its beginnings, cluster research continues to be an exciting area of science as most cluster properties remain much less known than those of their constituent atoms and molecules. Moreover, the scalability of clusters allows interpolation between the individual atom, the surface, and the bulk, bridging the gap between single atoms or molecules and condensed matter systems. This makes clusters unique targets to advance both the fundamental understanding of the many-body problem as well as nanotechnological applications [1-3].

Of particular interest are phenomena exhibiting clustersize dependence that underline the transition from individual atoms and molecules to large cluster systems with typical solid-state behavior, such as changes in cluster geometry and electronic structure. As each atom in the cluster is surrounded by an increasing number of neighbors, its electronic structure is altered by the resulting changes in the cluster potential and the atomic orbitals evolve into the band structure of the solid state. Cluster-size-dependent electronic properties can be probed directly using angle-resolved photoelectron spectroscopy, a well established technique for the study of the electronic structure of atoms, molecules, as well as condensed matter $[4,5]$. However, while photoelectron spectroscopy of clusters, and in particular rare-gas clusters, has been a growing field since the late 1980s [6-16], there is a paucity of angle-resolved measurements, mainly due to low target densities in the cluster beam and the resulting low signal intensities in angle-resolved measurements. To date, measurements of the photoelectron angular distribution parameter are only available for small metal clusters (e.g., [17]). For rare-gas clusters, recent qualitative studies by Öhrwall et al. [10] have shown substantial differences in the angular dependence of the photoelectron intensity from $\mathrm{Xe}$ clusters compared to free Xe atoms, but their experiment did not provide absolute measurements of the angular distribution parameter.

In this paper, we present the first quantitative measurement of the photoelectron angular distribution parameter $\beta$ [18] as a function of photon energy and cluster size for any rare-gas cluster system. Our experimental results are supported by multiple scattering calculations, which elucidate the effect of elastic electron scattering on the photoelectron angular distribution.

The measurements were carried out with linearly polarized synchrotron radiation from the undulator beamlines 8.0.1, 9.0.2, and 10.0.1 of the Advanced Light Source (ALS). A beam of rare-gas van der Waals clusters was produced by an adiabatic expansion cluster source and crossed with a beam of tunable synchrotron radiation (bandwidth $\approx 100-150 \mathrm{meV}$ ). For the present experiment, xenon gas with a stagnation pressure, $P_{0}=60-200 \mathrm{kPa}$, was expanded through a $100 \mu \mathrm{m}$ nozzle cooled to $170-240 \mathrm{~K}$ in order to produce clusters with average sizes $\langle N\rangle$ between 60 and 8000 atoms [19]. For the smaller clusters, up to $\langle N\rangle=1000$, a simple pinhole orifice was used, while the larger clusters were produced with a conical nozzle with half opening angle of $7^{\circ}$. The pinhole aperture has the advantage that the scaling laws used to determine the average cluster size are generally more accurate, but the maximum cluster size is limited by the high stagnation pressure required and the resulting load on the turbo pumps. A conical nozzle with small opening angle allows the production of much larger clusters, whose sizes, however, are known less accurately [12].

Photoelectrons were detected simultaneously in twoelectron time-of-flight (TOF) analyzers situated in the plane perpendicular to the light propagation direction at the "magic angle" $\left(54.7^{\circ}\right)$ and at $0^{\circ}$ with respect to the light polarization [20]. Simultaneous measurement at both angles is crucial for a quantitative determination of the photoelectron angular distribution parameter as it is independent of temperature and density fluctuations of the cluster beam. In order to achieve sufficient spectral resolution, suitable retarding voltages were applied to the drift tube of the TOF spectrometers. The photon energy was increased in small increments of 100-500 meV and each spectrum was recorded for $100 \mathrm{sec}-$ onds, resulting in a set of two-dimensional (2D) photoelectron maps [21] as shown in Fig. 1. These maps are ideally 


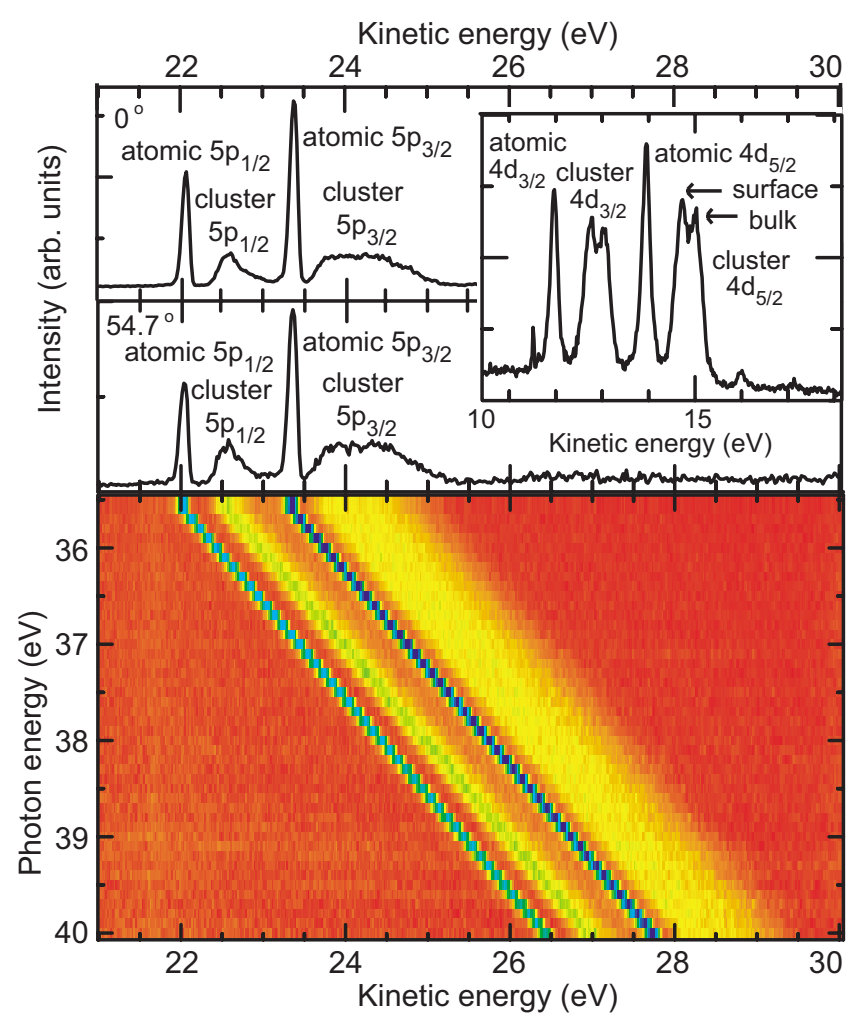

FIG. 1. (Color online) 2D photoelectron map for $5 p$ ionization of Xe clusters with average cluster size $\langle N\rangle=70$ measured with the "magic angle" analyzer $\left(54.7^{\circ}\right)$. Individual spectra illustrating the spin-orbit split photolines of the monomer as well as the cluster measured with $h \nu=35.5 \mathrm{eV}$ at $0^{\circ}$ and $54.7^{\circ}$ are shown above the 2D map. For comparison, the inset shows a $\mathrm{Xe} 4 d$ spectrum (average cluster size $\langle N\rangle=270$ ) measured at $h \nu=82.0 \mathrm{eV}$ with the $54.7^{\circ}$ analyzer.

suited for dealing with crowded spectra such as for Xe $4 d$ photoionization, where Auger lines often overlap with the photolines.

The top panels of Fig. 1 show photoelectron spectra of the Xe $5 p$ outer-valence shell measured at two different angles with respect to the light polarization direction. In addition to the two broad peaks, which correspond to the two spin-orbit components of the cluster photolines, the recorded spectra show a signal from free atoms (narrow peaks) also present in the gas beam. These atomic lines allow a direct comparison between the photoelectron angular distribution for the cluster and free atom. They also provide an accurate means to cali- brate the photoelectron kinetic energy and the relative efficiencies of the TOF analyzers using the well-known values for the atomic $5 p$ and $4 d$ ionization thresholds [22,23], as well as the atomic angular distribution parameters [24].

Compared to the much narrower $4 d$ cluster peaks with distinct surface and bulk components (see inset in Fig. 1), the $5 p$ cluster peaks are broad and structureless. This difference has been attributed to a stronger delocalization of the $5 p$ valence orbitals in contrast to the strongly localized $4 d$ core orbitals [13]. In this context, it is also worth noting the different widths of the $5 p_{1 / 2}$ and $5 p_{3 / 2}$ cluster lines, which are likely a result of the splitting of the $5 p_{3 / 2}$ into two bands due to crystal fields (see, e.g., [25]) and/or to direct bonding interactions between cluster atoms similar to the effect observed in thin Xe films [26].

Figure 2 compares the angular distribution parameters $\beta$ of the $\mathrm{Xe} 5 p$ and $4 d$ photoelectrons from small clusters $(\langle N\rangle=70-270)$, as a function of photon energy, to those of the free atoms. We find pronounced differences between the Xe $5 p$ cluster and atomic photoelectrons in the first $50 \mathrm{eV}$ above the $5 p$ threshold, with the angular distributions of the $5 p$ cluster photoelectrons being much more isotropic than the corresponding distributions of atomic $5 p$ photoelectrons. For higher photon energies, the angular distributions of $5 p$ cluster photoelectrons follow more closely those of free atoms, although the statistical error increases as the partial photoionization cross section and hence count rate rapidly decreases. In contrast to the large deviations observed for the $5 p$ electrons, the angular distributions for the $\mathrm{Xe} 4 d$ cluster and atomic photoelectrons are almost identical for these cluster sizes, except for very low kinetic energies, where the cluster photoelectrons are again more isotropic. A separate analysis of the angular distributions of surface and bulk components for this cluster size by means of a least-square fit [10] yields no difference within the experimental error, hence only the angular distributions of the total cluster peak intensity are shown in Fig. 2.

The cluster size dependence of the angular distribution parameter for Xe $4 d$ surface and bulk photoelectrons, measured at a photon energy $h \nu=150 \mathrm{eV}$, where the angular anisotropy is highest, is presented in Fig. 3. A significant decrease of the angular distribution parameter of the bulk component is observed for average cluster sizes larger than $\langle N\rangle=1000$, while the angular distribution of the surface component is only slightly smaller than the atomic value and stays constant within the range of the experimental error. These observations are consistent with the qualitative find-

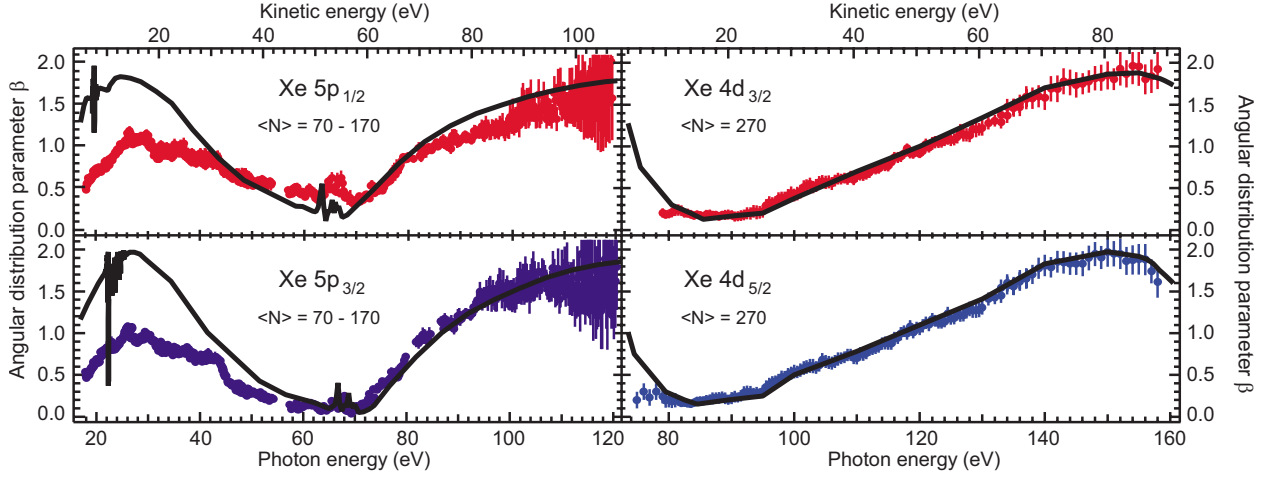

031201-2
FIG. 2. (Color online) Photoelectron angular distribution parameter $\beta$ of the spin-orbit components of the $\mathrm{Xe} 5 p$ and $4 d$ photolines in free clusters (circles) compared to single atoms (solid curves). Atomic values were taken from the literature [24] and were used to calibrate the relative spectrometer efficiencies. 


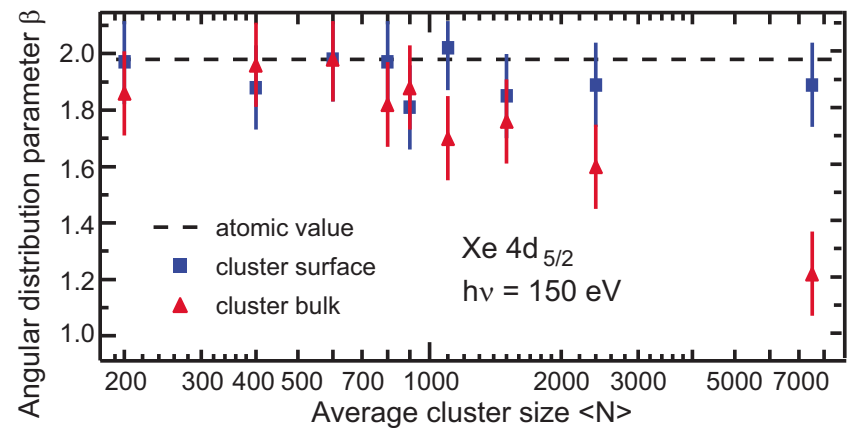

FIG. 3. (Color online) Xe $4 d$ photoelectron angular distribution parameter at $h \nu=150 \mathrm{eV}$ as a function of cluster size.

ings by Öhrwall et al., who reported a more isotropic angular distribution of photoelectrons from clusters with average sizes $\langle N\rangle=1000-4000$ compared to photoelectrons from free atoms, and also noted significant differences in the behavior of surface and bulk components [10]. This effect was attributed to elastic scattering of the photoelectrons by neighboring atoms in the cluster, leading to more isotropic angular distributions for electrons from the interior of the cluster than for those from the surface or a free atom.

In order to investigate the role of electron scattering in more detail, we have performed multiple scattering (MS) model calculations [27] and compare them to the experimental data in Fig. 4. The calculations start from atomic transition matrix elements, which reproduce the experimental angular distribution of atomic $5 p$ and $4 d$ photoelectrons as shown in Fig. 4. The cluster potential is treated in a muffintin approximation, and the results are averaged for random orientations of the cluster with respect to the incident light. In order to reduce the computation time, the calculations were performed for small clusters of 55 atoms in icosahedral geometry, and 53 atoms in a fcc structure (not shown here). To model the surface component, electron emission from the outermost layer was considered, while only electrons emitted from the innermost 10 atoms were taken for the bulk component. Our model calculations clearly reproduce the experi- mentally observed trends and confirm that the increased isotropy of the cluster photoelectrons can be attributed to elastic scattering of the ejected electrons by the neighboring atoms in the cluster. For the $4 d$, they seem to slightly overemphasize the effect of electron scattering and show deviations from the atomic distribution already for small clusters, where the experimental results are still close to the atomic value. A possible explanation may lie in an inadequate definition of bulk emitters in the calculation, where some intermediate atoms closer to the surface, whose electrons experience much less scattering, are left out. When extrapolating the calculated results to larger clusters, the finite escape depth of the photoelectrons also has to be taken into account. It effectively limits the influence of elastic scattering in larger clusters, since electrons emitted from the deepest atoms can no longer escape from the cluster $[12,28]$.

While the comparison of experimental data and model calculations clearly demonstrates the influence of elastic electron scattering on the photoelectron angular distribution, another intriguing effect, namely a possible connection to the geometric structure of the cluster, remains obscure. It is known that for several atomic and molecular clusters, a change from a noncrystalline (poly-)icosahedral structure to a crystalline fcc-lattice geometry occurs when the cluster reaches a critical size of several hundred to thousand atoms $[28,29]$. However, in the energy range considered here, the calculated difference in the angular distribution parameter for small clusters with icosahedral structure and fcc lattice is smaller than our experimental error. Additional studies are under way to explore this question further.

In conclusion, the examples of the $4 d$ and $5 p$ photoionization of Xe clusters presented in this work demonstrate that angle-resolved photoelectron spectroscopy is a sensitive probe of the electronic structure of van der Waals clusters. Our experimental methodology can be applied to any cluster system and thus represents a general technique to study the electronic structure of clusters. For the cases shown here, the angular distributions of the cluster photoelectrons are, in general, very similar to those of the corresponding independent atoms; however, distinct differences in the an-

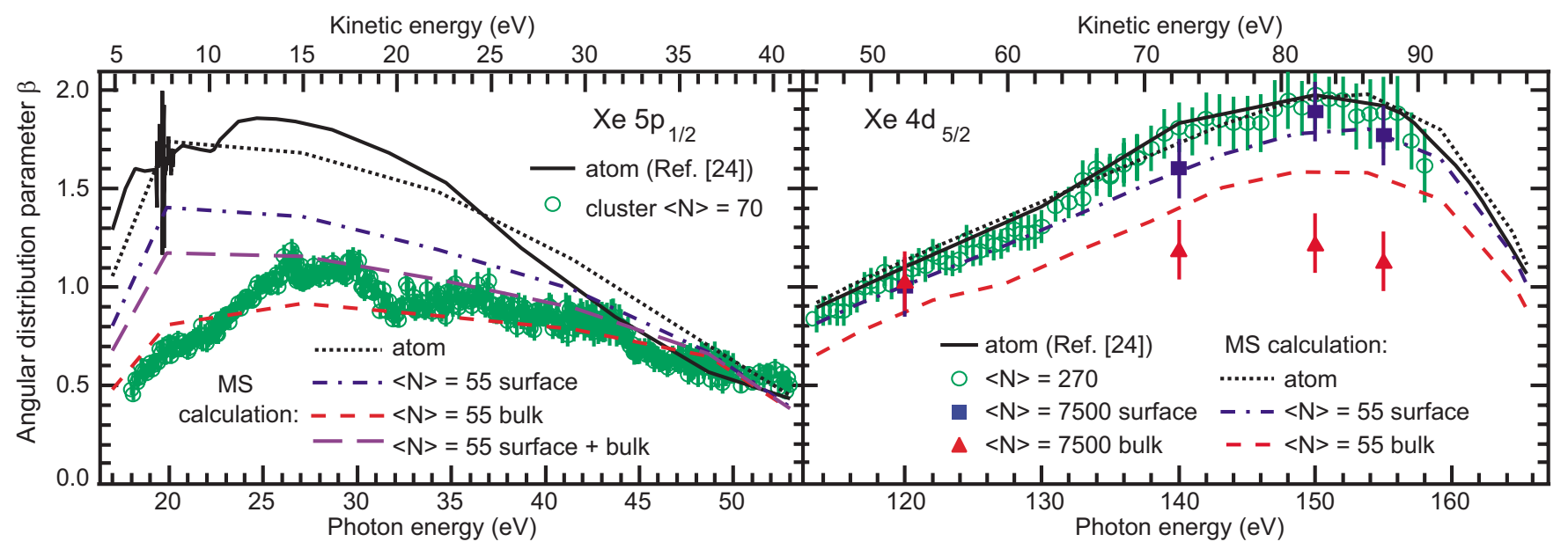

FIG. 4. (Color online) Experimental Xe $5 p$ and $4 d$ cluster photoelectron angular distribution parameter (symbols) compared to multiple scattering (MS) calculations for icosahedral clusters of 55 atoms (dashed and dash-dotted lines). The energy axis in the calculation is scaled to match calculated and experimental atomic $\beta$ curves (dotted and solid lines). 
gular distribution uncover cluster-size-dependent effects and, together with multiple scattering calculations, expose the role of elastic photoelectron scattering. While earlier measurements by Öhrwall et al. [10] did not allow a determination of the angular distribution parameter $\beta$, a qualitative agreement between the two experiments is found. Furthermore, our calculations show a small influence of the cluster structure on the photoelectron angular distribution, but the link to cluster geometry and electronic structure requires further experimental and theoretical elucidation. A deeper un- derstanding of cluster photoionization in comparison to similar studies on atoms and solids will shed new light on the closing gap between gas-phase and condensed matter physics.

The work was supported by the Office of Basic Energy Sciences, U.S. Department of Energy, Chemical Sciences, Geosciences and Biosciences Division. D.R. is grateful to the Alexander von Humboldt Foundation for support through the Feodor Lynen Program.
[1] Physics and Chemistry of Finite Systems: From Clusters to Crystals, edited by P. Jena, S. N. Khanna, and B. K. Rao (Kluwer Academic, Dordrecht, 1992), Vol. I.

[2] Clusters of Atoms and Molecules, edited by H. Haberland, Springer Series Chem. Physics Vol. 52 (Springer, Berlin, 1994-1995), Vols. I and II.

[3] Progress in Experimental and Theoretical Studies of Clusters, edited by T. Kondow and F. Mafune (World Scientific, Singapore, 2003).

[4] VUV and Soft X-Ray Photoionization, edited by U. Becker and D. A. Shirley (Plenum Press, New York, 1996), p. 135.

[5] Angle-Resolved Photoemission-Theory and Current Applications, edited by S. D. Kevan (Elsevier Science, Amsterdam, 1992).

[6] P. M. Dehmer and J. L. Dehmer, J. Chem. Phys. 67, 1774 (1977); 68, 3462 (1978); 69, 125 (1978).

[7] F. Carnovale, J. B. Peel, and R. G. Rothwell, J. Chem. Phys. 95, 1473 (1991).

[8] O. Björneholm, F. Federmann, F. Fössing, and T. Möller, Phys. Rev. Lett. 74, 3017 (1995); J. Chem. Phys. 104, 1846 (1996).

[9] U. Hergenhahn et al., Chem. Phys. Lett. 351, 235 (2002); S. Joshi, S. Barth, S. Marburger, V. Ulrich, and U. Hergenhahn, Phys. Rev. B 73, 235404 (2006).

[10] G. Öhrwall et al., J. Phys. B 36, 3937 (2003).

[11] D. S. Peterka, A. Lindinger, L. Poisson, M. Ahmed, and D. M. Neumark, Phys. Rev. Lett. 91, 043401 (2003).

[12] M. Tchaplyguine et al., J. Chem. Phys. 120, 345 (2004).

[13] R. Feifel et al., Eur. Phys. J. D 30, 343 (2004).

[14] A. Kivimäki et al., Phys. Rev. A 71, 033204 (2005).

[15] T. Hatsui et al., J. Chem. Phys. 123, 154304 (2005).

[16] M. Lundwall et al., Chem. Phys. Lett. 392, 433 (2004); J. Chem. Phys. 125, 014305 (2006); A. Lindblad et al., Phys.
Chem. Chem. Phys. 8, 1899 (2006).

[17] J. C. Pinaré, B. Baguenard, C. Bordas, and M. Broyer, Phys. Rev. Lett. 81, 2225 (1998).

[18] J. Cooper and R. N. Zare, J. Chem. Phys. 48, 942 (1968).

[19] R. Karnbach et al., Rev. Sci. Instrum. 64, 2838 (1993).

[20] N. Berrah et al., J. Electron Spectrosc. Relat. Phenom. 101103, 1 (1999).

[21] E. Sokell et al., J. Electron Spectrosc. Relat. Phenom. 94, 107 (1998).

[22] J. E. Hansen and W. Persson, Phys. Scr. 36, 602 (1987).

[23] G. C. King et al., J. Phys. B 10, 2479 (1977).

[24] M. O. Krause, T. A. Carlson, and P. R. Woodruff, Phys. Rev. A 24, 1374 (1981); S. H. Southworth et al., Nucl. Instrum. Methods Phys. Res. A 246, 782 (1986); M. G. Flemming, J. Z. Wu, C. D. Caldwell, and M. O. Krause, Phys. Rev. A 44, 1733 (1991); H. Wang et al., Phys. Rev. Lett. 87, 123004 (2001); O. Hemmers (private communication).

[25] N. Schwentner et al., Phys. Rev. Lett. 34, 528 (1975).

[26] K. Horn, M. Scheffler, and A. M. Bradshaw, Phys. Rev. Lett. 41, 822 (1978); T. Mandel, G. Kaindl, M. Domke, W. Fischer, and W. D. Schneider, ibid. 55, 1638 (1985); W. Widdra, Appl. Phys. A: Mater. Sci. Process. 72, 395 (2001).

[27] F. J. García de Abajo, M. A. Van Hove, and C. S. Fadley, Phys. Rev. B 63, 075404 (2001).

[28] F. G. Amar, J. Smaby, and T. J. Preston, J. Chem. Phys. 102, 2302 (2005).

[29] J. Farges et al., J. Chem. Phys. 78, 5067 (1983); 84, 3491 (1986); S. Goyal, D. L. Schutt, and G. Scoles, ibid. 102, 2302 (1995); S. Kakar et al., Phys. Rev. Lett. 78, 1675 (1997); J.-B. Maillet et al., Chem. Phys. 109, 329 (1998); 111, 2095 (1999); E. Rühl, Int. J. Mass. Spectrom. 229, 117 (2003), and references therein. 